\title{
Orthotopic liver transplantation (OLTx) in non-cirrhotic portal hypertension secondary to ADAMTS13 deficiency
}

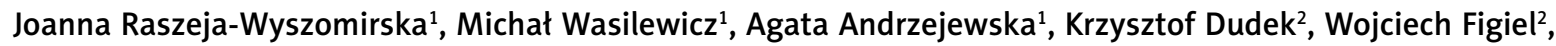 \\ Elżbieta Urasińska ${ }^{3}$, Bogna Ziarkiewicz-Wróblewska, Piotr Milkiewicz ${ }^{1}$, Marek Krawczyk² \\ ${ }^{1}$ Liver and Internal Medicine Unit, Department of General, Transplant, and Liver Surgery, Medical University of Warsaw, \\ Warsaw, Poland \\ 2Department of General, Transplant, and Liver Surgery, Medical University of Warsaw, Warsaw, Poland \\ ${ }^{3}$ Department of Pathomorphology, Pomeranian Medical University, Szczecin, Poland \\ ${ }^{4}$ Department of Pathology, Medical University of Warsaw, Warsaw, Poland
}

\begin{abstract}
Address for correspondence: Joanna Raszeja-Wyszomirska MD, Liver and Internal Medicine Unit, Department of General, Transplant, and Liver Surgery, Medical University of Warsaw, 1 A Banacha St, 02-097 Warsaw, Poland, phone: +48 225991662 , fax: +48 225991663 , e-mail: joanna.wyszomirska@wum.edu.pl
\end{abstract}

Non-cirrhotic intrahepatic portal hypertension $(\mathrm{NCIPH})$, also called idiopathic or benign, may lead to life-threatening complications. It is a rare indication for orthotopic liver transplantation (OLTx), although it may remain underdiagnosed. Overt symptoms of portal hypertension, predominantly variceal bleedings, misleadingly suggest that these patients are cirrhotic. Non-cirrhotic intrahepatic portal hypertension can be related to obliteration of portal venous microcirculation as a consequence of ADAMTS13 deficiency, a metalloproteinase which cleaves the ultra-large molecular weight forms of von Willebrand factor (VWF). The physiological role of VWF, secreted from endothelium, is to facilitate platelet adhesion at sites of endothelial damage. Decreased ADAMTS13 activity and persistence of ultra-large VWF at the endothelial surface predisposes to platelet clumping, causing microvascular occlusion. We describe the first OLTX for ADAMTS13 deficiency-related NCIPH in Poland.

A 20-year-old, previously healthy male student presented with upper gastrointestinal bleeding. Gastroduodenoscopy revealed active bleeding from oesophageal varices, which was successfully treated with endoscopic banding ligation. Physical examination showed splenomegaly, but was otherwise normal with no signs suggesting chronic liver disease. Laboratory investigations were all normal except thrombocytopenia of 40,000/ $\mathrm{ml}$. Viral, metabolic, and autoimmunological markers of liver disease as well as bone marrow examination were normal. Transjugular liver biopsy was performed and was essentially normal. However, the tissue sample size was rather slight and we were not able to measure hepatic venous pressure gradient (HVPG). Percutaneous liver biopsy performed later confirmed these findings. Contrast enhanced magnetic resonance imaging scan excluded portal vein thrombosis and Budd-Chiari syndrome. Laboratory investigations showed undetectable levels of ADAMTS13. The patient has been followed up over a period of 5 years, showing constant progression of his portal hypertension with continuous enlargement of his spleen (Figures 1 and 2) and frequent oesophageal/gastric variceal treatments. Follow-up liver biopsy showed progression to F2 fibrosis. He underwent OLTx with rapid recovery and remains extremely well 6 months after surgery. The histological changes in the explanted liver are shown in Figures $3 \mathrm{~A}-\mathrm{C}$.

Non-cirrhotic intrahepatic portal hypertension secondary to ADAMTS13 deficiency is a progressive condition and in case of uncontrolled symptoms of portal hypertension may require liver transplantation. Non-cirrhotic intrahepatic portal hypertension is also called idiopathic or benign portal hypertension. The latter term is misleading as it was shown that more than half of these patients develop liver failure when observed over a median period of 88 months [1]. Thus NCIPH is considered a rare but clinically important cause of portal hypertension. The underlying problem is related to the obliteration of portal venous microcirculation, secondary to the deficiency of ADAMTS13, a metalloproteinase 


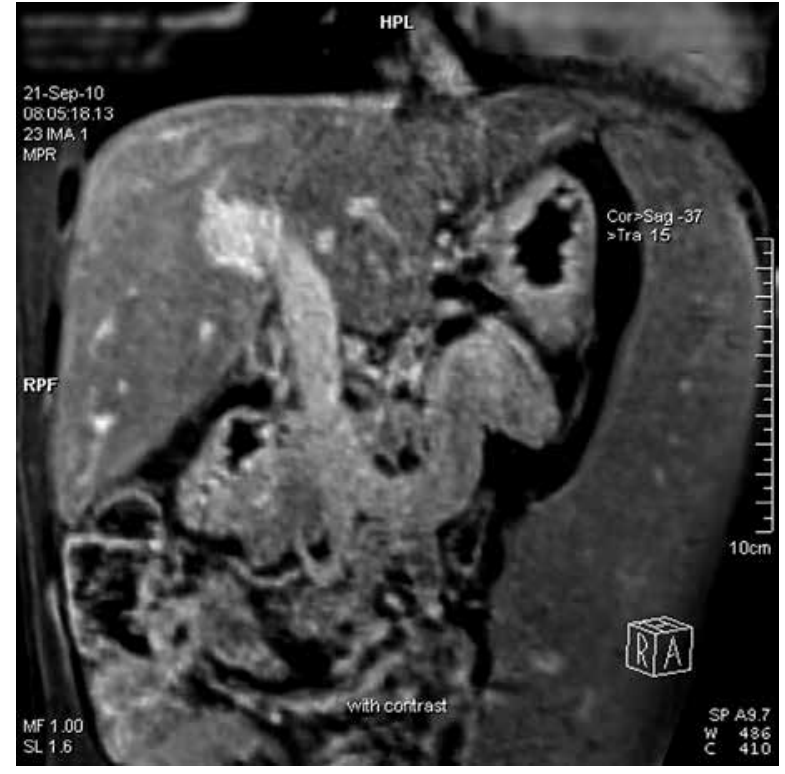

Figure 1. Magnetic resonance imaging of portal hypertension
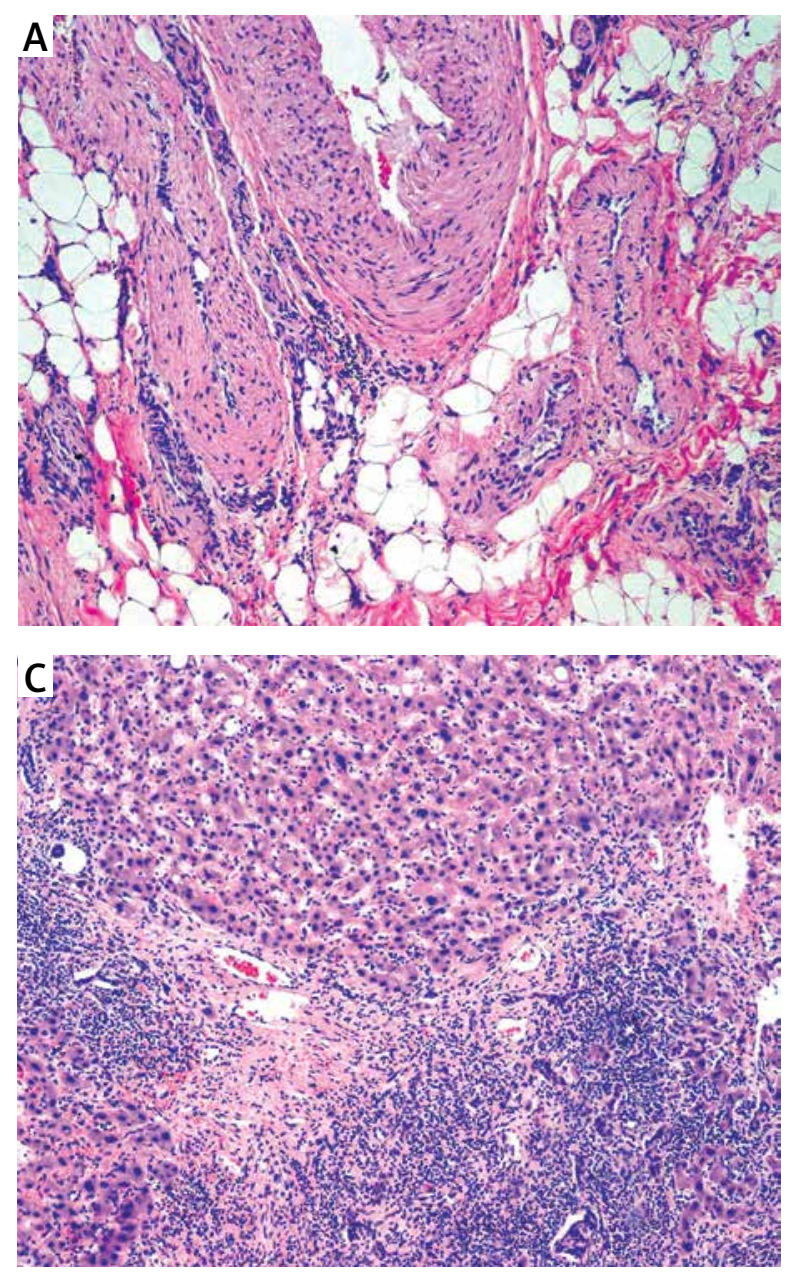

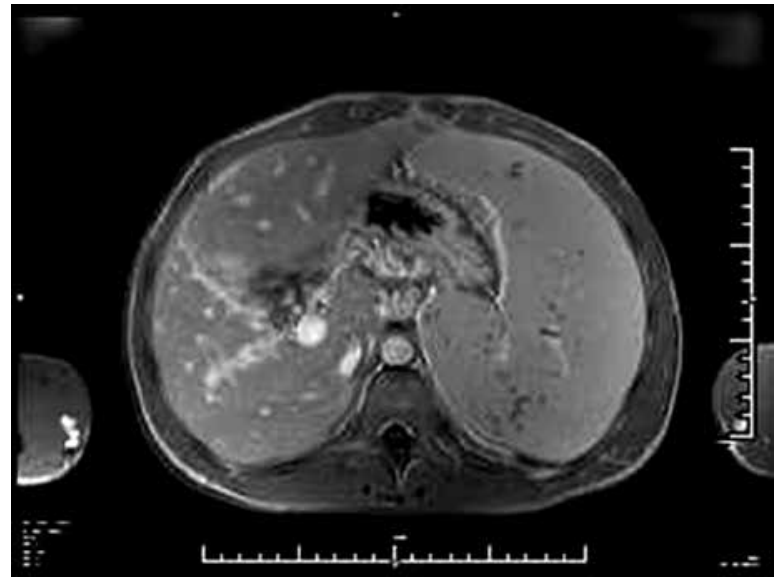

Figure 2. Computed tomography images of the liver hypotrophy and spleen enlargement

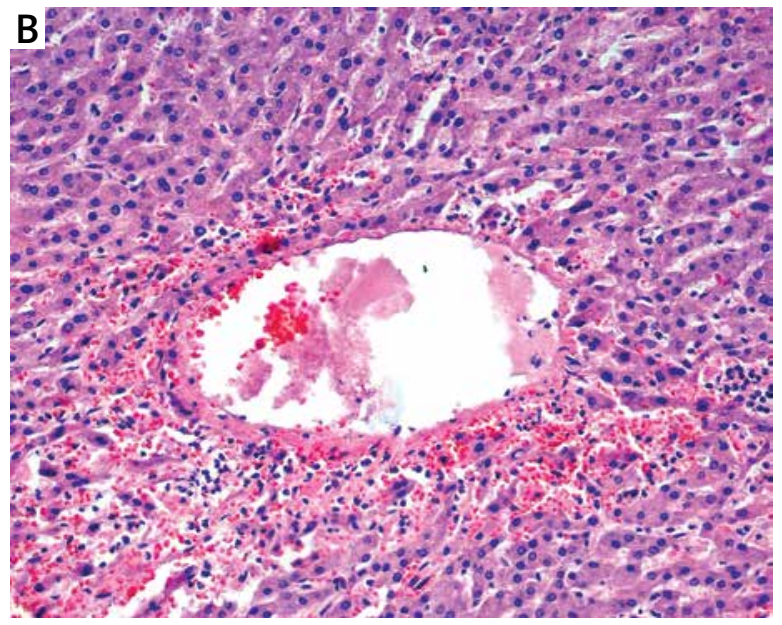

Figure 3. Histopathology of the explanted liver: A - Liver fibrosis with inflammatory infiltrates. Neocholangioles, small venules, and lack of correct arterioles. Haematoxylin and eosin stain $(\mathrm{H}+\mathrm{E})$. Objective magnification 10×. B - Thick irregular vessels with narrowed lumen in liver hilus. Haematoxylin and eosin stain $(\mathrm{H}+\mathrm{E})$. Objective magnification 4×. C - Venulitis, haemorrhages. Haematoxylin and eosin stain $(H+E)$. Objective magnification $20 x$ 
that cleaves the ultra-large molecular weight forms of VWF to smaller ones [2]. The physiological role of VWF, secreted from endothelium, is to facilitate platelet adhesion at sites of endothelial damage. Mutations of ADAMTS13 are seen in congenital TTP, while antibodies to ADAMTS13 are found in the majority of adult acquired cases. Decreased ADAMTS13 activity and persistence of ultra-large VWF at the endothelial surface is thought to predispose to platelet clumping, causing microvascular occlusion. The imbalance in VWF and ADAMTS13 levels in portal microcirculation, where hepatic arterial blood pressures are superimposed, with upstream relation to hepatic stellate cells, would provide a mechanism for obliteration of terminal portal venules, which is characteristic of NCIPH. In our patient plasma activity of ADAMTS13 was below $1 \%$, and its inhibitor was within the normal ranges. He has been treated with $\beta$-blockers and remains clinically stable with appropriate endoscopic surveillance. However, in sequential MRI and computed tomography imagines, atrophy of the right lobe of the liver has been observed, with enlargement of the left lobe, periportal fibrosis, varices, and dilatation of the portal tract veins. Chronic $\mathrm{NCIPH}$ leads to hepatic atrophy, although the progression of the disease is usually slow. The disorder can be associated with celiac disease and ulcerative colitis, and sustained deficiency of ADAMTS13 appears characteristic of NCIPH despite preserved liver function [1]. Older age at first presentation, hepatic encephalopathy, and portal vein thrombosis were found to be significant predictors of reduced transplant-free survival with, as already mentioned, progression to decompensated liver disease and the need for liver transplantation in a significant proportion of patients [1].

\section{Conflict of interest}

The authors declare no conflict of interest.

\section{References}

1. Eapen CE, Nightingale P, Hubscher SG, et al. Non-cirrhotic intrahepatic portal hypertension: associated gut disease and prognostic factors. Dig Dis Sci 2011; 56: 227-35.

2. Mackie I, Eapen CE, Neil D, et al. Idiopathic noncirrhotic intrahepatic portal hypertension is associated with sustained ADAMTS13 deficiency. Dig Dis Sci 2011; 56: 2456-65.

Received: 6.01 .2015

Accepted: 11.02 .2015 